\title{
Asymmetry in the Prediction of Cojumps on Volatility and Its Reversal
}

\author{
Liling Deng $\mathbb{D}^{1}{ }^{1}$ Zhiqiang Wang, $^{2}$ and Haifang Xiong ${ }^{2}$ \\ ${ }^{1}$ College of Finance and Economics, Chongqing Three Gorges University, Wanzhou 404130, China \\ ${ }^{2}$ College of Finance, Dongbei University of Finance and Economics, Dalian 116025, China \\ Correspondence should be addressed to Liling Deng; 20200007@sanxiau.edu.cn
}

Received 11 November 2021; Revised 8 December 2021; Accepted 9 December 2021; Published 2 March 2022

Academic Editor: Gengxin Sun

Copyright (c) 2022 Liling Deng et al. This is an open access article distributed under the Creative Commons Attribution License, which permits unrestricted use, distribution, and reproduction in any medium, provided the original work is properly cited.

The asymmetry of volatility is an important feature of asset price changes, and studying the asymmetry of cojumps on volatility prediction can provide a reference for systemic risk monitoring. Based on high-frequency data of CSI 300 stock index and futures index, SSE Composite Index, and industry index, the influence of cojumps on volatility in different directions is analyzed by HAR model. It is found that the explanation of volatility by cojumps still has leverage effect, and the symbolic cojumps covariance can obviously improve the prediction ability of the model; influenced by policy reform and market changes, the prediction of cojumps on volatility between the CSI 300 stock index and futures index shows a significant interval reversal effect; not only is the prediction of systematic cojumps on volatility of each industry index related to the periodicity of the industry, but also it shows different rules under the influence of policy reform.

\section{Introduction}

Asset price volatility has been a core variable in financial fields such as risk management, investment, and pricing. With the rapid development of computer and communication technology, the prediction of realized volatility (RV) and the capture of price jumps under high frequency data have become a hot topic of research for scholars at home and abroad. Under the trend of financial market integration, the risk spillover mechanism and price linkage reaction among markets have led to the gradual attention to price cojumps between different markets or assets. Barndorff-Nielsen and Shephard separated the cojumps component from the quadratic covariance for the first time and established the metric [1]. Under the efficient market hypothesis, cojumps are usually more intuitive in response to macro information shocks, and studying the characteristics of cojumps and their prediction of volatility can not only inform the monitoring of systemic risk but also provide guidance to investors for optimal decision-making and risk aversion.

Numerous studies have shown the importance of cojumps for the prediction of covariance models. Gilder et al. conducted statistics on the jumps of broad market index and the covariance between their components and found that the two have a high overlap probability and concluded that there is a close relationship between systematic cojumps and macro information release [2]. Wang et al. constructed the Jump Covariance (JCOV) factor and found that this factor was able to significantly improve the prediction accuracy of the binary HAR model [3]. Tang and Lin studied the performance of major domestic indexes such as the Shenzhen Composite Index and SSE Composite Index and found that their jumps directions were basically the same during the sample period, the frequency of cojumps was higher, and the introduction of cojumps variation could improve the prediction of multiasset HAR-RCV and HARRCV-CJ models [4]. Qu and Ji found that incorporating the cojumps factor into the HAR model can effectively improve the prediction of covariance from the point of view of cojumps intensity [5].

A typical feature of asset price volatility is the leverage effect, whereby prices typically react more strongly to bad news than to good news, a phenomenon also known as asymmetry in volatility. This asymmetry places more 
emphasis on the impact of extreme returns, that is, jumps on volatility. By constructing monthly jumps indicators under high-frequency data, Zuo and Liu found that negative jumps can reflect the asymmetry of volatility more clearly by constructing monthly jump index under highfrequency data and improve the prediction effect of volatility [6]. Zhao and Qin verified that macro information shocks have a significant effect on jumps and further found that positive (negative) shocks have a decreasing (increasing) effect on the probability of stock price jumps [7]. Patton and Sheppard put positive the concept of symbol jump variation according to the difference of jump direction and pointed out that the distinction of jump direction symbols can improve the prediction ability of HAR model [8]. The research results of Pu et al. on Chinese stock market showed that the sign jump variance can bring better out-of-sample forecasting ability and economic value to HAR models compared to GARCH-type models, and the impact of negative sign jumps was greater than that of positive sign jumps [9]. Ma et al. evaluated the forecasting performance of HAR class models in a new symbolic returns framework, and the study showed that the models that include symbolic jumps and symbolic returns have optimal out-of-sample forecasting accuracy. Based on the correction for realized volatility, the adjusted extended realized jump variance is closer to the true level [10]. It can be seen that, at present, the research on asymmetry at home and abroad is mostly related to the jump category of a single asset, but the research on the cojumps characteristics of multiple assets is rare.

In addition, if individual stocks and the index jump at the same time, it is a systematic jump and vice versa for heterogeneous jump. As most stocks usually show the direction characteristics of "rising and falling with the same price" when fluctuating with the market, further research on the asymmetry of systemic jumps to volatility prediction has important reference value for studying the time-varying characteristics of systemic risk. Bollerslev et al. constructed an equal weight combination of 30 weighted stocks and found that there is a significant systemic jumps behavior in the American stock market under the mcp test based on the mean-cross product method [11]. Zhou and Zhu found that systematic jumps are important pricing factors in the return model in the study of the domestic market (Shanghai Stock Exchange), and, for some securities portfolios, this component can indeed improve the prediction of the model [12]. Evidence for the existence of systematic jumps was derived by Jian and $\mathrm{Li}$ in their discussion on the time-varying characteristics of beta coefficients [13], and Liu et al. decomposed the risk characteristics of jumps according to the index-stock method. It was also found that the influence of the right tail jumps is greater than that of the left tail jumps [14]. Again, it can be seen that, due to the abovementioned limitations of studies on asymmetry, there are almost no studies on systematic cojumping. In fact, systematic cojumps outperform systematic jumps in terms of both time properties and information scope and provide a relatively more comprehensive picture of the extent of systematic risk shocks.
In summary, the current research on the characteristics of individual asset jumps is more mature, while the depth of research on multiasset cojumps is relatively lacking. Not only has the literature failed to consider the influence of leverage effect when analyzing the prediction of cojumps on volatility, but also the research objects are mostly limited to some parallel markets (such as stock index and futures index, Shanghai Composite Index, Shenzhen Composite Index, etc.), and there is no in-depth analysis of the systematic factors involving the composite index and individual stocks (or local index), which to a certain extent affect the accuracy and reliability of the relevant conclusions. Based on the above analysis, this paper considers introducing leverage effect and sign cojumps factors into the HAR volatility model and further investigates the prediction properties of systematic cojumps. Its innovations are mainly reflected in three aspects discussed as follows.

First, the direction sign of cojumps is considered in the volatility research, and whether cojumps also use the prediction of volatility is tested.

Second, by constructing a new symbol cojumping estimator-symbol covariance, the significance of adding an asymmetry factor to the volatility model from the perspective of out-of-sample prediction is revealed.

Third, under the framework of asymmetry in volatility, we further examine the impact of coexistence with systemic risk characteristics on future volatility and then analyze the risk characteristics and cyclical attributes of various industries in the market.

\section{Variable Selection and Model Setting}

2.1. Tests for Cojumps. For the identification of cojumps, this paper adopts the synchronization occurrence criterion [15] proposed by Lahaye et al., that is, to determine the cohop of multiple assets according to the intersection principle on the basis of identifying a single jump. Among them, the jumps test of individual assets mainly depends on the S-BNS method of Barndorff-Nielsen and Shephard and the BTL criterion proposed by Bollerslev et al. [16].

Assuming that the price is measurable in interval $(0,1,2, \ldots, M, \ldots, M T)$, the logarithmic rate of return of the price in interval $(t-1, t)$ can be expressed as

$$
r_{t, i}=P_{t, i+1}-P_{t, i} \quad t=1,2, \ldots, T, i=1,2, \ldots, M,
$$

where $T \in N$ is the number of days and $M \in N$ is the number of intervals of intraday high-frequency sampling. Then realized volatility and realized double power variation can be expressed as follows:

$$
\begin{aligned}
& R V_{t}=\sum_{i=1}^{M}\left|r_{t, i}\right|^{2}, \\
& B V_{t}=\frac{\pi}{2} \sum_{i=1}^{M}\left|r_{t, i}\right|\left|r_{t, i-1}\right| .
\end{aligned}
$$

First, the BTL criterion defines a random pattern of intraday fluctuations as 
$\mathrm{TOD}_{i}=\frac{(M+1) \sum_{t=1}^{T}\left|r_{t, i}\right|^{2} I\left(\left|r_{t, i}\right| \leq \tau \sqrt{B V_{t} \wedge R V_{t}} M^{-\bar{\omega}}\right)}{\sum_{t=1}^{T} \sum_{i=1}^{M}\left|r_{t, i}\right|^{2} I\left(\left|r_{t, i}\right| \leq \tau \sqrt{B V_{t} \wedge R V_{t}} M^{-\bar{\omega}}\right)}$,

where $\omega \in(0,0.5), \tau>0$, and generally let $\tau=2.5$ and $\varpi=0.49$. The truncated variance constructed by the above parameters enables a more intuitive identification of highfrequency jumps. According to the BTL method, the jumps variable at time $i$ on day $t$ is

$$
\mathrm{Jump}_{t, i}=r_{t, i} I\left(\left|r_{t, i}\right| \geq \tau \sqrt{B V_{t} \wedge R V_{t} * \mathrm{TOD}_{i}} M^{-\bar{\omega}}\right)
$$

Also, the BNS test statistic selected for this paper is

$$
Z_{t}-\log -i m=\frac{\log \left(R V_{t}\right)-\log \left(B V_{t}\right)}{\sqrt{\left((\pi / 2)^{2}+\pi-5\right) 1 / M \max \left(1, \mathrm{TPV} / \mathrm{TBPV}_{t}^{2}\right)}}
$$

In the formula,

$$
\begin{aligned}
\mathrm{TBPV}_{t} & =\mu_{4 / 3}^{-3} \sum_{i=1}^{M-2} \omega_{i}\left|r_{t, i}\right|^{4 / 3}\left|r_{t, i+1}\right|^{4 / 3}\left|r_{t, i+2}\right|^{4 / 3}, \\
\omega_{i} & =\frac{\left(\sum_{t} \sum_{i=1}^{M-2}\left|r_{t, i}\right|\left|r_{t, i+1}\right|^{2}\right)}{(M-2) \sum_{t}\left|r_{t, i}\right|^{4 / 3}\left|r_{t, i+1}\right|^{4 / 3}\left|r_{t, i+2}\right|^{4 / 3}}, \quad i=1,2, \ldots, M-2 .
\end{aligned}
$$

Then the jumps variable at time $i$ on day $t$ under the S-BNS method is

$$
\operatorname{Jump}_{t, i}=r_{t, i} I\left(r_{t, i}=\max _{1 \leq j \leq M} r_{t, j}\right) I\left(Z_{t}-\log -i m>\Phi_{1-\alpha}^{-1}\right) .
$$

In order to improve the efficiency of identifying real jumps, this paper chooses to use the BTL criterion in overnight periods and the joint test of the S-BNS criterion for cojumps in intraday periods according to the TOD setting.

2.2. Directional Symbol for Cojumps. According to Wang et al. [3], the variable to measure the cojumps information between the stock index and the futures index on day $t$ is defined as

$$
\mathrm{JCOV}_{t}=\sum_{i=1}^{M} \mathrm{Jump}_{t, i}^{S} \mathrm{Jump}_{t, i}^{F},
$$

where Jump $\mathrm{Ju}_{t, i}^{S}$ and Jump $\mathrm{J}_{t, i}^{F}$ represent the jumps in the stock and futures index, respectively.

In the actual market, the probability of the stock index and the futures index making opposite jumps at the same moment is almost zero. Therefore, the positive and negative cojumps covariances are defined separately as follows:

$$
\begin{aligned}
& \operatorname{JCOV}_{t}^{+}=\sum_{k=1}^{M} \operatorname{Jump}_{t, k}^{S} \operatorname{Jump}_{t, k}^{F} I\left(\operatorname{Jump}_{t, k}^{S}>0, \operatorname{Jump}_{t, k}^{F}>0\right), \\
& \operatorname{JCOV}_{t}^{-}=\sum_{k=1}^{M} \operatorname{Jump}_{t, k}^{S} \operatorname{Jump}_{t, k}^{F} I\left(\operatorname{Jump}_{t, k}^{S}<0, \operatorname{Jump}_{t, k}^{F}<0\right) .
\end{aligned}
$$

Since cojumps can be explained to a greater extent by shocks to historical information releases, the direction of cojumps corresponds to the nature of the information itself, and positive and negative cojumps covariance can correspond to the impact of positive versus negative news, respectively.

2.3. Systematic Cojumps. To study the systematic factors of covariance on volatility prediction, this paper selects the SSE Composite Index and its six representative sector indices, SSE material, energy, telecommunication, financial, consumption, and medicine, as the research subjects, at which point the cojumps covariance represents a measure of systematic risk for each sector index. The cojumps covariance between the SSE Composite Index and the sector indices is noted as

$$
\operatorname{JCOV}_{t}=\sum_{i=1}^{M} \operatorname{Jump}_{t, i}^{0} \mathrm{Jump}_{t, i}^{j}
$$

where Jump $\mathrm{Ju}_{t, i}^{S}$ and Jump $\mathrm{Ju}_{t, i}^{F}$ represent the jump covariates of the SSE Composite Index and each sector index, $j=1,2, \ldots, 6$. Similarly, the positive and negative systematic cojumps covariances are defined as follows:

$$
\begin{aligned}
\operatorname{JCOV}_{t}^{+} & =\sum_{k=1}^{M} \operatorname{Jump}_{t, k}^{0} \operatorname{Jump}_{t, k}^{j} I\left(\operatorname{Jump}_{t, k}^{0}>0, \operatorname{Jump}_{t, k}^{j}>0\right), \\
\mathrm{JCOV}_{t}^{-} & =\sum_{k=1}^{M} \operatorname{Jump}_{t, k}^{0} \operatorname{Jump}_{t, k}^{j} I\left(\operatorname{Jump}_{t, k}^{0}<0, \operatorname{Jump}_{2} \frac{1^{j}, k}{2}<0\right) .
\end{aligned}
$$

2.4. HAR Model Setting. Corsi proposed that the Heterogeneous Autoregressive (HAR) model not only has better out-of-sample prediction ability but also has a simpler form than the ARFIMA model, which can describe the long memory and thick tail of financial returns [17]. Most of the studies on cojumps and leverage effects in the literature have also been based on the HAR-RV model [18-20]. In this paper, we choose its logarithmic form: 
$\log \left(R \widehat{V_{t, t+1}}\right)=\alpha_{0}+\alpha_{d} \widehat{R V_{t}}+\alpha_{w} R \widehat{V_{t-5, t}}+\alpha_{m} R \widehat{V_{t-22, t}}+\varepsilon_{t+1}$,

where $\quad X_{t, t+1} \equiv\left(X_{t+1}+X_{t+2}+\cdots+X_{t+l}\right) / l, \quad l=1,5,22$, and $X_{t}, X_{t-5}$, and $X_{t-22}$ represent the daily, weekly, and monthly cumulative average estimates for the lagged period, respectively.

The modelling variables $\widehat{R V}$ are chosen in the form of an all-day weighted realized volatility that combines intraday effects with overnight factors:

$$
\widehat{R V_{t}}=\lambda_{t} \mathrm{WRVD}_{t}
$$

where $\mathrm{WRVD}_{t}=\sum_{i=2}^{M} \omega_{i} r_{t, i}^{2}$ is the weighted intraday realized volatility and $\omega_{i}$ and $\lambda_{t}$ are the weighting factor and overnight factor, respectively, defined as follows:

$$
\begin{aligned}
& \omega_{i}=\frac{\sum_{t} \sum_{i=2}^{M} r_{t, i}^{2}}{M \sum_{t} r_{t, i}^{2}}, \\
& \lambda_{t}=\frac{\sum_{k=t-m-1}^{t}\left(r_{k, n}^{2}+r_{k, d}^{2}\right)}{\sum_{k=t-m-1}^{t} r_{k, d}^{2}},
\end{aligned}
$$

where $r_{t, n}^{2}$ and $r_{t, d}^{2}$ represent the intraday volatility and overnight volatility and $m$ is the length of the historical moving window for predicting $\lambda_{t} ; m=22$ is selected in this paper to set the parameters corresponding to the length of the month.

To investigate the effect of cojumps factors on the prediction model, the HAR-RV-JCOV model was first developed based on Wang et al. [3].

$$
\begin{aligned}
\log \left(R \widehat{V_{t, t+1}}\right)= & \alpha_{0}+\alpha_{d} \widehat{R V_{t}}+\alpha_{w} R \widehat{V_{t-5, t}} \\
& +\alpha_{m} R \widehat{V_{t-22, t}}+\alpha_{j} \mathrm{JCOV}_{t}+\varepsilon_{t+1} .
\end{aligned}
$$

A further decomposition of JCOV yields the HAR-RVJCOV-D model with respect to asymmetry.

$$
\begin{aligned}
\log \left(R \widehat{V_{t, t+1}}\right)= & \alpha_{0}+\alpha_{d} \widehat{R V_{t}}+\alpha_{w} R \widehat{V_{t-5, t}} \\
& +\alpha_{m} R \widehat{V_{t-22, t}}+\alpha_{j_{1}} \mathrm{JCOV}_{t}^{+}+\alpha_{j 2} \mathrm{JCOV}_{t}^{-}+\varepsilon_{t+1} .
\end{aligned}
$$

'The identification of systematic cojumps and the development of the corresponding HAR-RV-JCOV-D model are an application of the asymmetry of volatility in the framework of the decomposition of risk characteristics.

\section{The Leverage Effect of Cojumps}

\subsection{Descriptive Statistical Characteristics of the Data}

3.1.1. Statistical Characteristics of Jumps and Cojumps. The sample selected in this section is 5-minute high-frequency data for the CSI 300 stock and futures index markets from January 7, 2013, to January 19, 2018. Table 1 provides the statistical characteristics of the price jumps and cojumps in the stock and futures index markets during the sample period. It can be seen that the number of days in which jumps occur in the CSI 300 futures index is greater than that in the stock index, and the mean (absolute value) and standard deviation of futures jumps are greater than those of spot jumps, regardless of positive or negative jumps, indicating that the futures market is more volatile and has a higher degree of risk. On the one hand, futures trading implements a margin system, and this special trading mechanism has a leveraging effect that amplifies returns while also amplifying risks; on the other hand, the positive nature of futures contracts determines the increase in future uncertainties, and various factors affecting the spot market may also lead to volatility in the futures market. Secondly, although the frequency of jumps themselves is not high, the proportion of cojumps in the total jumps, that is, the frequency of cojumps, is not low (in the stock index and futures market, the frequency of cojumps reaches $42.011 \%$ and $42.127 \%$, respectively), which indicates that the occurrence of cojumps is not a coincidental phenomenon, and there is a practical basis for studying the factor reflecting the information of cojumps as a separate influencing factor.

\subsubsection{Descriptive Statistical Characteristics of the Cojumps} Covariance. Since the covariance has the possibility of taking zero value, $\log (\mathrm{JCOV}+1)$ is expressed in the model in logarithmic form. It can be seen from Table 2 that each logarithmic covariance sequence rejects the null hypothesis of normal distribution, and the "spikes" and "heavy tails" are more prominent, and they show significant autocorrelation. At the same time, the ADF statistics show that the series are all smooth. The parameters are estimated using ordinary least squares (OLS) combined with Newey-West covariance correction.

\subsection{Asymmetry in the Prediction of Cojumps on Volatility}

3.2.1. Analysis of the Leverage Effect of Cojumps. From Table 3, it is not difficult to find that, whether in the stock index or futures index market, the fitting accuracy of the model has been significantly improved after adding cojumps covariance; after further decomposing JCOV, it is found that the fitting accuracy of the HAR-WRVH-JCOV-D model also increases, indicating that the cojumps components in different directions have different explanatory validity for volatility, and the constraints imposing on the coefficients are unfavourable for the prediction of volatility At the same time, the coefficient $\alpha_{j_{2}}$ of negative cojumps is consistently statistically significantly positive, indicating that the effect of negative cojumps is greater than that of positive cojumps. Overall, therefore, the prediction of cojumps on volatility is also significantly leveraged, which corresponds to the need to be cautious about some bearish macro policy making and information releases in practice to prevent excessive shocks to the market and triggering financial panic.

3.2.2. Interval Reversal Analysis of the Leverage Effect. Generally, changes in macro information and economic policies will directly affect investors' expectations and trigger 
TABLE 1: Statistical characteristics of jumps and cojumps.

\begin{tabular}{|c|c|c|c|}
\hline & Spot jumps & Futures jumps & Cojumps \\
\hline \multicolumn{4}{|l|}{ All jumps } \\
\hline Total observation numbers & 58176 & 58176 & 58176 \\
\hline Jumps days & 437 & 518 & 287 \\
\hline Jumps times & 726 & 724 & 305 \\
\hline Average value & 0.010 & 0.011 & 0.002 \\
\hline Standard deviation & 0.228 & 0.251 & 0.011 \\
\hline Jumps probability (\%) & 1.248 & 1.244 & 0.524 \\
\hline Cojumps probability (\%) & 42.011 & 42.127 & 41.026 \\
\hline \multicolumn{4}{|l|}{ Positive jumps } \\
\hline Jumps days & 246 & 343 & 155 \\
\hline Jumps times & 379 & 426 & 164 \\
\hline Average value & 0.008 & 0.010 & 0.002 \\
\hline Standard deviation & 0.111 & 0.171 & 0.023 \\
\hline Jumps probability (\%) & 0.651 & 0.732 & 0.282 \\
\hline Cojumps probability (\%) & 43.272 & 38.498 & 37.861 \\
\hline \multicolumn{4}{|l|}{ Reverse jumps } \\
\hline Jumps days & 244 & 236 & 132 \\
\hline Jumps times & 347 & 298 & 141 \\
\hline Average value & 0.010 & 0.011 & 0.004 \\
\hline Standard deviation & 0.183 & 0.170 & 0.028 \\
\hline Jumps probability (\%) & 0.596 & 0.512 & 0.242 \\
\hline Cojumps probability (\%) & 40.634 & 47.315 & 38.217 \\
\hline
\end{tabular}

Jumps probability $=$ jumps times/total observation numbers $* 100$; cojumps probability $=$ cojumps times/jumps times $* 100$.

TABLE 2: Descriptive statistical characteristics of covariance of cojumps.

\begin{tabular}{lccc}
\hline LOG $(+1)$ & JCOV & JCOV+ & JCOV- \\
\hline Mean & -6.878297 & -6.895864 & -6.890095 \\
Max & -5.120204 & -5.193353 & -5.120204 \\
Min & -6.999377 & -6.907755 & -6.907755 \\
Std. dev & 0.135385 & 0.075106 & 0.114341 \\
Skewness & 9.096642 & 15.444781 & 11.216561 \\
Kurtosis & 98.845932 & 297.621003 & 146.063904 \\
J-B & $454849.2^{*}$ & $4193991^{*}$ & $1002217^{*}$ \\
ADF & $-16.35581^{*}$ & $21.524^{*}$ & $-19.86904^{*}$ \\
Q(5) & $154.221^{*}$ & $22.186^{*}$ & $44.609^{*}$ \\
Q(10) & $161.711^{*}$ & $22.186^{*}$ & $50.674^{*}$ \\
Q(20) & $168.362^{*}$ & $25.766^{*}$ & $56.737^{*}$ \\
\hline
\end{tabular}

${ }^{*},{ }^{* *}$, and ${ }^{* * *}$ represent significance at the $1 \%, 5 \%$, and $10 \%$ levels, respectively.

TABLE 3: The estimation of HAR-RV-JCOV-D model.

\begin{tabular}{|c|c|c|c|c|c|c|c|}
\hline & $\alpha_{d}$ & $\alpha_{w}$ & $\alpha_{m}$ & $\alpha_{j}$ & $\alpha_{j_{1}}$ & $\alpha_{j_{2}}$ & $R^{2}(\%)$ \\
\hline MS & $0.4481^{*}(8.9860)$ & $0.4809^{*}(10.0063)$ & $-0.9357^{* *}(-2.1456)$ & & & & 75.99 \\
\hline MJS & $0.4442 *(9.0093)$ & $0.4733^{*}(9.8989)$ & $-0.9750^{* *}(-2.2977)$ & $0.4205^{*}(3.5723)$ & & & 76.20 \\
\hline MJDS & $*(8.9$ & $0.4769^{*}(9.87$ & $-1.0142^{* *}(-2.4074)$ & & $0.0884(0.4985)$ & $0.5597^{*}(4.4456)$ & 76.44 \\
\hline MF & $0.2865^{*}(6.1448)$ & $0.6425^{*}(13.5888)$ & $98(-1.2$ & & & & 72.14 \\
\hline MJF & $0.2741^{*}(6.0532)$ & $0.6427^{*}(13.7113)$ & $-0.5720(-1.3135)$ & $0.4073^{* *}(2.3320)$ & & & 72.31 \\
\hline MJDF & $0.2724^{*}(5.9461)$ & $0.6481^{*}(13.5848)$ & $-0.5886(-1.3584)$ & & $-0.0635(-0.4400)$ & $0.6004^{*}(3.4683)$ & 72.59 \\
\hline
\end{tabular}

MS, MJS, and MJDS represent HAR-RV, HAR-RV-JCOV, and HAR-RV-JCOV-D models for the CSI 300 stock index, respectively, while MF, MJF, and MJDF represent the HAR-RV, HAR-RV-JCOV, and HAR-RV-JCOV-D models of the CSI 300 stock index, respectively, and ${ }^{*},{ }^{* *}$, and ${ }^{* * *}$ represent significance at the $1 \%, 5 \%$, and $10 \%$ levels, respectively.

market trends. At the same time, under different quotations and environments, investors' risk appetite and attitude may be completely different. Therefore, based on the demarcation point between policy and market changes, this article further specifically studies the asymmetric characteristics of market fluctuations at different stages and analyzes the possibility of interval reversals in different environments.

(1) Bounded by the Policy-Supply-Side Reform. Since the subprime crisis, the basic framework of China's macrocontrol has been mainly the combination of active fiscal 
policy and loose monetary policy, which promotes economic growth through demand-side management, resulting in a large area of traditional overcapacity and resource oversupply. On the other hand, the effective supply of emerging industries appears to be relatively insufficient. At the end of 2015, the Central Economic Work Conference stressed the promotion of supply-side structural reform and proposed reducing factor costs, alleviating overcapacity, and increasing the supply of emerging industries. The policy focus has gradually shifted from the traditional short-term stimulus and demand management to the pattern of "grasping both supply and demand," which is bound to have a great impact on the economic development cycle and the fluctuations of the financial market. Therefore, this paper makes a stage analysis of the samples according to the fluctuation characteristics before and after the supply-side reform. For the CSI 300 stock index and futures index market, due to the lack of data trading days, the first stage before the supply-side reform is from March 14, 2013, to January 28,2016 , and the second stage after the supply-side reform is from January 29, 2016, to January 19, 2018. For the Shanghai Composite Index, the first stage before the supplyside reform is from March 14, 2013, to January 26, 2016, and the second stage after the supply-side reform is from January 27, 2016, to January 19, 2018.

As can be seen from Table 4, volatility still maintains some long memory characteristics across the intervals. Although the coefficient of the weekly effect $\left(\alpha_{w}\right)$ before the supply-side reform is still greater than the daily effect $\left(\alpha_{d}\right)$, the situation is different after the reform, when $\alpha_{w}$ is less than $\alpha_{d}$ for the stock market. The end of 2015 coincided with the end of the stock market crash; the policy tightening directly led to a decline in initial production, a sharp drop in market volatility, and the economy entered a stage of recession; from 2016 to 2017, the prices of some upstream raw materials began to rise, but the economy was still in the stage of recovery. Although the stock market has picked up somewhat, the price volatility has been greatly weakened compared with before, and the duration of the fluctuation has also become significantly shorter.

For the CSI 300 stock index market, the coefficients measuring volatility asymmetry $\alpha_{j 1}$ and $\alpha_{j 2}$ have different statistical significance in the two stages. In the first stage, coefficient $\alpha_{j 2}$ is significantly positive, and $\alpha_{j 1}$ is not statistically significant, while in the second stage the conclusion is the opposite, indicating that, with the adjustment of the relationship between actual volatility and market expectations before and after the reform, the leverage effect of cojumps on the volatility of the stock index market has reversed direction, and the existence of this reversal effect indicates that China's stock market has gradually tended to develop and mature, and the investment concept and rational degree of investors are becoming more and more perfect.

As for the CSI 300 futures index, although volatility tends to moderate in general after the supply-side reform, it still maintains a high persistence in a certain period of time, and the weekly effect coefficient $\left(\alpha_{w}\right)$ remains larger than the daily effect coefficient $\left(\alpha_{d}\right)$; at the same time, the intensity of the shock brought by the reform is smaller than that of the stock index market, and the change in volatility is basically within the elastic range of market expectations, so the futures index market before and after the supply-side reform reversal effect is not obvious.

(2) Bounded by the Market-Bull and Bear Markets. During mid-to-late 2014 and 2015, the entire capital market experienced an alternating bull and bear market boom and collapse. Due to factors such as investor structure, expectations, and risk preferences, the leverage effect is prone to take different forms in extreme environments such as bull and bear markets. $\mathrm{Lu}$ and $\mathrm{Xu}$ confirmed that there is a significant leverage effect in China's stock market based on the E-GARCH model, and the forms of leverage effect during the bull market and bear market are not the same. Based on the characteristics of return movements, this paper further distinguishes the first stage into two intervals of bull and bear markets and investigates the response characteristics of volatility in different stages, respectively. For the CSI 300 stock index and futures index, the sample interval with bull market characteristics is selected from April 9, 2014, to May 31,2015 , and the sample interval with bear market characteristics is selected from June 1, 2015, to January 28, 2016.

As a whole, no matter the bull market or bear market, the impact of negative cojumps is significant in the index market, and the characteristics of high leverage and high risk determine that most investors have risk aversion attitude and stop-loss psychology. As can be seen from Table 5, the interpretation of future volatility by cojumps has different degrees of asymmetry in both stock and futures markets, and this asymmetric effect is more obvious during bear markets; and when the market switches from bull to bear markets, the direction of the leverage effect reverses very significantly, where the coefficient of the effect of positive cojumps $\alpha_{j 1}$ even changes from a significantly positive value to a significantly negative value. During the bear market, the economy enters a period of recession and adjustment, the stock market is at a historical low, the volatility of the stock market is less than the market expectation, and the positive cojumps can significantly enhance the future volatility. At this time, the good news can cause a greater market reaction, that is, the interval reversal of leverage effect.

The final comparison is with the jumps model. According to the definition of signed jumps (SJ) variation and the convergence property, the positive jumps variation $\left(J V_{t}^{+}\right)$and negative jumps variation $\left(J V_{t}^{-}\right)$are defined as follows:

$$
\begin{aligned}
& J V_{t}^{+}=\frac{J V_{t}+S J_{t}}{2}, \\
& J V_{t}^{-}=\frac{J V_{t}-S J_{t}}{2},
\end{aligned}
$$

where $J V_{t}=R V_{t}-B V_{t}$ and $J V_{t}$ and $S J_{t}$ are the jumps variance and sign jumps variance, respectively. Based on the sign definition of the jumps variance, the HAR-RV-JV-D model is developed as follows:

$$
\begin{aligned}
R V_{t+1}= & \gamma_{0}+\gamma_{d} B V_{t}+\gamma_{w} B V_{t-5, t}+\gamma_{m} B V_{t-22, t} \\
& +\gamma_{j 1} J V_{t}^{+}+\gamma_{j 2} \mathrm{CJV}_{t}^{-}+\varepsilon_{t+1} .
\end{aligned}
$$


TABLE 4: The estimation of HAR-RV-JCOV-D model based on policy demarcation.

\begin{tabular}{|c|c|c|c|c|c|c|}
\hline & $\alpha_{d}$ & $\alpha_{w}$ & $\alpha_{m}$ & $\alpha_{j_{1}}$ & $\alpha_{j_{2}}$ & $R^{2}(\%)$ \\
\hline \multicolumn{7}{|c|}{ First phase } \\
\hline MJDS & $0.3393^{*}(6.1027)$ & $0.5695^{*}(10.3700)$ & $-0.3079(-0.8807)$ & $-0.0743(-0.5922)$ & $0.5910^{*}(4.5360)$ & 74.58 \\
\hline MJDF & $0.1693^{*}(2.8518)$ & $0.7201^{*}(11.5982)$ & $0.6145^{* * *}(1.6923)$ & $-0.1081(-0.8139)$ & $0.6398^{*}(3.5412)$ & 76.57 \\
\hline \multicolumn{7}{|c|}{ Phase II } \\
\hline $\begin{array}{l}\text { MJDS } \\
\text { MJDF }\end{array}$ & $\begin{array}{l}0.5236^{*}(7.0948) \\
0.3643^{*}(5.6933)\end{array}$ & $\begin{array}{l}0.2709^{*}(3.7670) \\
0.4357^{*}(5.6978)\end{array}$ & $\begin{array}{c}-1.5753^{* * *}(-2.0896) \\
-2.4191^{*}(-3.0024)\end{array}$ & $\begin{array}{c}3.8236^{*}(3.4609) \\
0.4643(0.3007)\end{array}$ & $\begin{array}{l}-0.9015(-1.2498) \\
-0.5111(-0.6683)\end{array}$ & $\begin{array}{l}54.08 \\
46.23\end{array}$ \\
\hline
\end{tabular}

Note: MJDS and MJDF represent HAR-RV-JCOV-D models for the CSI 300 stock index and futures index, respectively, and ${ }^{*},{ }^{* *}$, and ${ }^{* * *}$ represent significance at the $1 \%, 5 \%$, and $10 \%$ levels, respectively.

TABLE 5: The estimation of HAR-RV-JCOV-D model based on market boundary.

\begin{tabular}{|c|c|c|c|c|c|c|}
\hline & $\alpha_{d}$ & $\alpha_{w}$ & $\alpha_{m}$ & $\alpha_{j_{1}}$ & $\alpha_{j_{2}}$ & $R^{2}(\%)$ \\
\hline \multicolumn{7}{|c|}{ Bull market } \\
\hline MJDS & $0.2895^{* *}(2.5677)$ & $0.6242 *(5.1835)$ & $-0.5482(-1.0711)$ & $1.3150^{* *}(2.0272)$ & $0.8510^{*}(4.3897)$ & 76.20 \\
\hline MJDF & $0.1871^{* * *}(1.9315)$ & $0.6780 *(6.1020)$ & $0.4904(0.7484)$ & $1.0303^{* * *}(1.8857)$ & $0.8701^{*}(4.7913)$ & 66.11 \\
\hline \multicolumn{7}{|c|}{ Bear market } \\
\hline MJDS & $0.3647^{*}(3.5029)$ & $0.4195^{*}(4.4671)$ & $0.7208(1.0713)$ & $-0.0847(-0.7442)$ & $0.4616^{* *}(2.4861)$ & 56.68 \\
\hline MJDF & $0.2932^{*}(2.6460)$ & $0.5410^{*}(4.7227)$ & $0.9210(1.4457)$ & $-0.2038^{* * *}(-1.8285)$ & $0.4685^{* *}(2.3628)$ & 63.68 \\
\hline
\end{tabular}

Note: MJDS and MJDF represent HAR-RV-JCOV-D models for the CSI 300 stock index and futures index, respectively, and ${ }^{*},{ }^{* *}$, and ${ }^{* * *}$ represent significance at the $1 \%, 5 \%$, and $10 \%$ levels, respectively.

Comparing Tables 5 and 6 , it is not difficult to find that the reversal characteristics of cojumps are not the same as those of jumps in the futures index market. The leverage effect on jumps is unaffected by changes in market sentiment and always remains constant in the same direction, while the leverage effect on cojumps reverses more significantly when the market switches from a bullish to a bearish environment. The data show that positive jumps in the futures index have almost no effect on volatility, while positive cojumps in the stock index and futures index show significant effects in bullish forecasts, suggesting that there is a certain degree of spillover between the two markets and that information shocks in the stock index market are likely to be transmitted to the futures market and that this transmission is mainly in the form of positive jumps.

3.3. Symbolic Cojumps Covariance. Based on the idea of constructing the sign jumps variance, the positive symbolic cojumps covariance (SJCOV+) and the negative symbolic cojumps covariance (SJCOV-) are defined, respectively, for the CSI 300 stock index and the futures market as follows:

$$
\begin{aligned}
& \operatorname{SJCOV}_{t}^{+}=\sqrt{\operatorname{SJVs}_{t}^{+} \mathrm{SJVF}_{t}^{+}}, \\
& \operatorname{SJCOV}_{t}^{+}=\sqrt{\mathrm{SJVs}_{t}^{-} \mathrm{SJVF}_{t}^{-}} .
\end{aligned}
$$

For the SSE sector indices, the symbolic cojumps covariance is defined as

$$
\begin{aligned}
\operatorname{SJCOV}_{t, j}^{+} & =\sqrt{\mathrm{SJVO}_{t}^{+} \mathrm{SJV}_{t}^{+}}, \\
\operatorname{SJCOV}_{t}^{+} & =\sqrt{\mathrm{SJVO}_{t}^{-} \mathrm{SJV}_{t}^{-}} .
\end{aligned}
$$

where SJVS ${ }_{t}$ and $\mathrm{SJVF}_{t}$ represent the sign jumps variance of the stock index and the futures index, respectively, and $S J V 0_{t}$ and and $\mathrm{SJV}_{t, j}$ represent the sign jumps variance of the SSE
Composite Index and each sector index, respectively, $j=1,2, \ldots, 6$. According to the above definition method, SJCOV assigns different signs to the cojumps in different directions, SJCOV+represents the positive cojumps of the day, and SJCOV- represents the negative cojumps of the day.

Symbolic cojumps covariance is introduced into the HARRV and HAR-RV-JCOV models, and the HAR-RV-SJCOV-D and HAR-RV-JCOV-SJCOV-D models are obtained:

$$
\begin{aligned}
\mathrm{WRVH}_{t+1}= & \alpha_{0}+\alpha_{d} \mathrm{WRVH}_{t}+\alpha_{w} \mathrm{WRVH}_{t-5, t} \\
& +\alpha_{m} \mathrm{WRVH}_{t-22, t}+\beta_{j 1} \mathrm{SJCOV}_{t}^{+}+\beta_{j 2} \mathrm{SJCOV}_{t}^{-} \\
& +\varepsilon_{t+1}, \\
\mathrm{WRVH}_{t+1}= & \alpha_{0}+\alpha_{d} \mathrm{WRVH}_{t}+\alpha_{w} \mathrm{WRVH}_{t-5, t} \\
& +\alpha_{m} \mathrm{WRVH}_{t-22, t}+\alpha_{j} \mathrm{JCOV}_{t}+\beta_{j 1} \mathrm{SJCOV}_{t}^{+} \\
& +\beta_{j 2} \mathrm{SJCOV}_{t}^{-}+\varepsilon_{t+1} .
\end{aligned}
$$

According to the results in Tables 7 and 8, the in-sample accuracy of the model is significantly improved by adding the symbolic cojumps covariance to the CSI 300 stock index, the futures index, and the SSE sector index; at the same time, $\beta_{j 2}$ almost uniformly shows a significantly positive number, which indicates that the symbolic cojumps covariance also has a significant asymmetric property in the prediction of volatility, and the effect of negative cojumps covariance plays a leading role.

Next, SPA tests were performed on the prediction accuracy of the two sets of models, HAR-RV (HAR-RV-JCOV) and HAR-RV-SJCOV-D (HAR-RV-JCOV-SJCOV-D), respectively. In turn, one group is set as the benchmark model $\left(R_{0}^{2}\right)$ and the other group is the model to be tested, and the out-of-sample prediction accuracy of the model to be tested and the benchmark model is evaluated by calculating the $p$ value corresponding to the SPA statistic under the six types 
TABLE 6: Estimation results of HAR-RV-JV-D model considering market boundaries.

\begin{tabular}{|c|c|c|c|c|c|c|}
\hline & $\gamma_{d}$ & $\gamma_{w}$ & $\gamma_{m}$ & $\gamma_{j_{1}}$ & $\gamma_{j_{2}}$ & $R^{2}(\%)$ \\
\hline \multicolumn{7}{|c|}{ Bull market } \\
\hline MJDS & $0.3331^{*}(3.4066)$ & $0.5426^{*}(5.1643)$ & $-0.9790^{* * *}(-1.9388)$ & $5.2725^{*}(2.9206)$ & $0.7809^{*}(4.2862)$ & 77.70 \\
\hline MJDF & $0.2250^{* *}(2.0879)$ & $0.6524^{*}(5.6169)$ & $-0.4012(-0.5368)$ & $0.0880(0.2344)$ & $0.7562^{* *}(2.1457)$ & 65.63 \\
\hline \multicolumn{7}{|c|}{ Bear market } \\
\hline MJDS & $0.1768^{* * *}(1.7099)$ & $0.5224^{*}(5.0602)$ & $1.5572^{* * *}(1.9155)$ & $0.0170(0.0493)$ & $0.8007^{*}(6.0153)$ & 54.86 \\
\hline MJDF & $0.2576^{* *}(2.2550)$ & $0.5820^{*}(4.9564)$ & $0.2577(0.4790)$ & $-0.2465^{* * *}(-1.8243)$ & $0.4723^{*}(3.6473)$ & 62.80 \\
\hline
\end{tabular}

Note: MJDS and MJDF represent HAR-RV-JCOV-D models for the CSI 300 stock index and futures index, respectively, and ${ }^{*},{ }^{* *}$, and ${ }^{* * *}$ represent significance at the $1 \%, 5 \%$, and $10 \%$ levels, respectively.

TABLE 7: HAR-RV-SJCOV-D model parameter estimation results.

\begin{tabular}{|c|c|c|c|c|c|c|c|}
\hline & $\alpha_{d}$ & $\alpha_{w}$ & $\alpha_{m}$ & $\beta_{j 1}$ & $\beta_{j 2}$ & $R^{2}(\%)$ & $R_{0}^{2}(\%)$ \\
\hline MSJS & $0.4107^{*}(8.1612)$ & $0.4960^{*}(10.3464)$ & $-1.0678^{* *}(-2.4199)$ & $-1.7831(-1.2487)$ & $8.5850(3.4918)$ & 76.39 & 75.99 \\
\hline MSJF & $0.2655^{*}(5.6821)$ & $0.6432 *(13.8702)$ & $-0.8441^{* * *}(-1.9121)$ & $-2.9128(-1.4391)$ & $10.2950^{*}(4.3666)$ & 72.75 & 72.14 \\
\hline MSJMA & $0.5005^{*}(12.5364)$ & $0.4028^{*}(9.7122)$ & $-0.2632(-0.6959)$ & $1.4611(0.7463)$ & $7.3349^{*}(3.4730)$ & 73.82 & 73.51 \\
\hline MSJEL & $0.4763^{*}(10.1210)$ & $0.4225^{*}(9.3792)$ & $-1.7562^{*}(-3.8770)$ & $0.1262(0.0634)$ & $6.8121^{*}(3.3899)$ & 74.63 & 74.26 \\
\hline MSJFI & $0.3660 *(8.0568)$ & $0.5321^{*}(11.7360)$ & $-0.7651^{* * *}(-1.8742)$ & $0.0330(0.0174)$ & $13.3071^{*}(4.8638)$ & 73.40 & 72.88 \\
\hline MSJEN & $0.4216^{*}(8.2480)$ & $0.4667^{*}(9.6814)$ & $-0.9969^{*}(-2.7386)$ & $1.8958(0.9404)$ & $6.4216^{*}(3.0110)$ & 71.25 & 71.05 \\
\hline MSJCO & $0.4552 *(8.9773)$ & $0.4268^{*}(8.5645)$ & $-1.4559^{*}(-3.5269)$ & $2.1232(1.3942)$ & $7.7856^{*}(3.7348)$ & 71.46 & 71.16 \\
\hline MSJME & $0.4535^{*}(9.7469)$ & $0.4575^{*}(10.2969)$ & $-1.5962^{*}(-3.4077)$ & $-0.7180(-0.2507)$ & $7.0815^{* *}(2.2716)$ & 75.11 & 74.92 \\
\hline
\end{tabular}

Note: MSJS and MSJF represent the HAR-RV-SJCOV-D models for the CSI 300 stock and futures index, respectively; MSJMA, MSJEL, MSJFI, MSJEN, MSJCO, and MSJME represent the HAR-RV-SJCOV-D model of material, telecommunication, finance, energy, consumption, and medicine, respectively. $R_{0}^{2}$ denotes the HAR-RV model's adjusted goodness of fit, and ${ }^{*},{ }^{* *}$, and ${ }^{* * *}$ represent significance at the $1 \%, 5 \%$, and $10 \%$ levels, respectively.

TABLE 8: Results of parameter estimation of HAR-WRVH-JCOV-SJCOV-D model.

\begin{tabular}{|c|c|c|c|c|c|c|c|c|}
\hline & $\alpha_{d}$ & $\alpha_{w}$ & $\alpha_{m}$ & $\alpha_{j}$ & $\beta_{j 1}$ & $\beta_{j 2}$ & $R^{2}(\%)$ & $R_{0}^{2}(\%)$ \\
\hline MJSJS & $\begin{array}{l}0.4075^{*} \\
(8.2102)\end{array}$ & $\begin{array}{c}0.4884^{*} \\
(10.2240)\end{array}$ & $\begin{array}{c}-1.1042^{* *} \\
(-2.5731)\end{array}$ & $0.4088^{*}(3.7466)$ & $\begin{array}{c}-1.7641 \\
(-1.2639)\end{array}$ & $\begin{array}{l}8.4623^{*} \\
(3.5694)\end{array}$ & 76.59 & 76.20 \\
\hline MJSJF & $\begin{array}{l}0.2538^{*} \\
(5.6012)\end{array}$ & $\begin{array}{c}0.6433^{*} \\
(13.9344)\end{array}$ & $\begin{array}{c}-0.8553^{* * *} \\
(-1.9428)\end{array}$ & $\begin{array}{c}0.3966^{* *} \\
(2.3721)\end{array}$ & $\begin{array}{l}-2.7839 \\
(-1.3210)\end{array}$ & $\begin{array}{c}10.2756^{*} \\
(4.5565)\end{array}$ & 72.91 & 72.31 \\
\hline MJSJMA & $\begin{array}{c}0.4968^{*} \\
(12.4562)\end{array}$ & $\begin{array}{l}0.3929^{*} \\
(9.5774)\end{array}$ & $-0.2693(-0.7230)$ & $\begin{array}{c}0.3153^{* * *} \\
(1.9903)\end{array}$ & $1.3712(0.6949)$ & $7.1215^{*}(3.3952)$ & 73.93 & 73.64 \\
\hline MJSJEL & $\begin{array}{c}0.4764^{*} \\
(10.1889)\end{array}$ & $\begin{array}{c}0.4144^{*} \\
(9.2588)\end{array}$ & $-1.7519^{*}(-3.8817)$ & $0.1772(1.2962)$ & $0.0356(0.0171)$ & $\begin{array}{l}6.6605^{*} \\
(3.3494)\end{array}$ & 74.65 & 74.31 \\
\hline MJSJFI & $\begin{array}{l}0.3635^{*} \\
(8.0640)\end{array}$ & $\begin{array}{c}0.5265^{*} \\
(11.6744)\end{array}$ & $\begin{array}{c}-0.7717^{* * *} \\
(-1.9038)\end{array}$ & $0.4802^{*}(4.4605)$ & $\begin{array}{c}-0.4129 \\
(-0.2143)\end{array}$ & $\begin{array}{l}11.8221^{*} \\
(4.5098)\end{array}$ & 73.58 & 73.19 \\
\hline MJSJEN & $\begin{array}{c}0.4178^{*} \\
(8.2299)\end{array}$ & $\begin{array}{l}0.4570^{*} \\
(9.6344)\end{array}$ & $-0.9490^{*}(-2.5945)$ & $0.4334^{*}(3.4521)$ & $1.9323(0.9987)$ & $\begin{array}{l}5.9543^{*} \\
(2.8473)\end{array}$ & 71.48 & 71.28 \\
\hline MJSJCO & $0.4532 *(89462)$ & $\begin{array}{l}0.4221^{*} \\
(8.4206)\end{array}$ & $-1.4429^{*}(-3.5124)$ & $0.1834(1.0723)$ & $2,1721(1.4491)$ & $7.7883^{*}(3.8071)$ & 71.47 & 71.17 \\
\hline MJSJME & $\begin{array}{l}0.4616^{*} \\
(9.6030)\end{array}$ & $\begin{array}{l}0.4417^{*} \\
(9.5387)\end{array}$ & $-1.5962^{*}(-3.4094)$ & $\begin{array}{l}0.4531^{* *} \\
(2.3820)\end{array}$ & $\begin{array}{c}-1.0869 \\
(-0.3929)\end{array}$ & $\begin{array}{c}6.3769^{* *} \\
(2.0161)\end{array}$ & 75.24 & 75.09 \\
\hline
\end{tabular}

Note: MSJS and MSJF represent the HAR-RV-SJCOV-D models for the CSI 300 stock and futures index, respectively; MSJMA, MSJEL, MSJFI, MSJEN, MSJCO, and MSJME represent the HAR-RV-SJCOV-D model of material, telecommunication, financial, energy, consumption, and medicine, respectively. $R_{0}^{2}$ denotes the HAR-RV model's adjusted goodness of fit, and ${ }^{*},{ }^{* *}$, and ${ }^{* * *}$ represent significance at the $1 \%, 5 \%$, and $10 \%$ levels, respectively.

of loss functions. The larger the $p$ value of the benchmark model relative to the to-be-examined model under a certain loss function criterion, the higher the prediction accuracy of that model.

According to the test results in Tables 9 and 10, the $p$ values of the HAR-WRVH-SJCOV-D (HAR-WRVHJCOV-SJCOV-D) group models for the CSI 300 stock index and futures index markets are consistently larger than those of the HAR-WRVH (HAR-WRVH-JCOV) group models without the addition of symbolic cojumps under the six loss function criteria such as MSE. In particular, in the stock index market, the $p$ values obtained when using HARWRVH-SJCOV-D (HAR-WRVH-JCOV-SJCOV-D) as the benchmark model are much larger than those of the other group, indicating that the introduction of symbolic cojumps covariance can indeed improve the prediction accuracy of the volatility model. For each industry index, similar conclusions are obtained for more than four loss function standards, except for indices such as medicine and telecommunication, which have more pronounced noncyclical 
TABLE 9: SPA test for HAR-RV and HAR-RV-SJCOV-D control models.

\begin{tabular}{|c|c|c|c|c|c|c|}
\hline Loss function & MSE & MAE & HMSE & HMAE & QLIKE & RLOG \\
\hline \multicolumn{7}{|l|}{ Base model } \\
\hline MSJS & 0.745 & 0.773 & 0.918 & 0.887 & 0.794 & 0.822 \\
\hline MS & 0.244 & 0.210 & 0.102 & 0.125 & 0.218 & 0.173 \\
\hline MSJF & 0.511 & 0.469 & 0.472 & 0.458 & 0.501 & 0.483 \\
\hline MF & 0.008 & 0.094 & 0.001 & 0.077 & 0.002 & 0.007 \\
\hline MSJMA & 0.894 & 0.114 & 0.559 & 0.207 & 0.591 & 0.598 \\
\hline MMA & 0.108 & 0.898 & 0.057 & 0.782 & 0.098 & 0.064 \\
\hline MSJEL & 0.462 & 0.080 & 0.494 & 0.163 & 0.578 & 0.676 \\
\hline MEL & 0.550 & 0.549 & 0.079 & 0.849 & 0.468 & 0.297 \\
\hline MSJFI & 0.733 & 0.255 & 0.744 & 0.307 & 0.799 & 0.791 \\
\hline MFI & 0.276 & 0.755 & 0.230 & 0.713 & 0.203 & 0.211 \\
\hline MSJEN & 0.471 & 0.904 & 0.480 & 0.933 & 0.452 & 0.470 \\
\hline MEN & 0.045 & 0.080 & 0.012 & 0.050 & 0.025 & 0.013 \\
\hline MSJCO & 0.621 & 0.069 & 0.638 & 0.124 & 0.629 & 0.755 \\
\hline MCO & 0.405 & 0.588 & 0.046 & 0.873 & 0.383 & 0.224 \\
\hline MSJME & 0.000 & 0.000 & 0.000 & 0.000 & 0.000 & 0.000 \\
\hline MME & 0.226 & 0.259 & 0.203 & 0.261 & 0.228 & 0.223 \\
\hline
\end{tabular}

Note: MS, MF, MMA, MEL, MFI, MEN, MCO, and MME represent the HAR-RV models for the CSI 300 stock index, the futures index, and the material, telecommunication, financial, energy, consumption, and medicine indices, respectively, and MSJS, MSJF, MSJMA, MSJEL, MSJFI, MSJEN, MSJCO, and MSJME represent the CSI 300 HAR-RV-JCOV-SJCOV-D models for stock, futures, and sector indices of material, telecommunication, financial, energy, consumption, and medicine, respectively, and the figures in the table represent the $p$ values of the SPA tests.

TABLE 10: SPA test for HAR-RV-JCOV and HAR-RV-JCOV-SJCOV-D control models.

\begin{tabular}{|c|c|c|c|c|c|c|}
\hline Loss function & MSE & MAE & HMSE & HMAE & QLIKE & RLOG \\
\hline \multicolumn{7}{|l|}{ Base model } \\
\hline MJSJS & 0.677 & 0.754 & 0.856 & 0.875 & 0.679 & 0.752 \\
\hline MJS & 0.322 & 0.260 & 0.118 & 0.146 & 0.291 & 0.252 \\
\hline MJSJF & 0.543 & 0.805 & 0.466 & 0.397 & 0.517 & 0.547 \\
\hline MJF & 0.009 & 0.198 & 0.005 & 0.159 & 0.013 & 0.008 \\
\hline MJSJMA & 0.847 & 0.094 & 0.532 & 0.155 & 0.893 & 0.570 \\
\hline MJMA & 0.136 & 0.923 & 0.065 & 0.817 & 0.101 & 0.087 \\
\hline MJSJEL & 0.389 & 0.123 & 0.503 & 0.255 & 0.455 & 0.580 \\
\hline MJEL & 0.605 & 0.506 & 0.105 & 0.770 & 0.533 & 0.363 \\
\hline MJSJFI & 0.783 & 0.383 & 0.788 & 0.488 & 0.861 & 0.810 \\
\hline MJFI & 0.181 & 0.618 & 0.206 & 0.496 & 0.157 & 0.166 \\
\hline MJSJEN & 0.449 & 0.787 & 0.478 & 0.866 & 0.471 & 0.463 \\
\hline MJEN & 0.068 & 0.188 & 0.011 & 0.102 & 0.054 & 0.051 \\
\hline MJSJCO & 0.508 & 0.069 & 0.613 & 0.0101 & 0.504 & 0.677 \\
\hline MJCO & 0.514 & 0.609 & 0.039 & 0.883 & 0.485 & 0.306 \\
\hline MJSJME & 0.000 & 0.000 & 0.000 & 0.000 & 0.000 & 0.000 \\
\hline MJME & 0.245 & 0.228 & 0.232 & 0.263 & 0.240 & 0.200 \\
\hline
\end{tabular}

Note: MS, MF, MMA, MEL, MFI, MEN, MCO, and MME represent the HAR-RV models for the CSI 300 stock index, the futures index, and the material, telecommunication, financial, energy, consumption, and medicine indices, respectively, and MSJS, MSJF, MSJMA, MSJEL, MSJFI, MSJEN, MSJCO, and MSJME represent the CSI 300 HAR-RV-JCOV-SJCOV-D models for stock, futures, and sector indices of material, telecommunication, financial, energy, consumption, and medicines, respectively, and the figures in the table represent the $p$ values of the SPA tests.

characteristics. Thus, the symbolic cojumps covariance not only has an asymmetric impact on volatility forecasting but also can significantly improve the goodness-of-fit and outof-sample forecasting ability of the model, which can better portray and predict the volatility conditions and risk characteristics of the Chinese financial market.

\section{The Prediction of Systematic Cojumps on Volatility}

4.1. Descriptive Statistical Characteristics of the Data. The sample in this section is the 5-minute high-frequency data for the SSE Composite Index and its sector index from January 7, 2013, to January 19, 2018. According to Table 11, each logarithmic cojumps covariance series satisfies the conditions for building the model and performing OLS estimation. When the subject of study is the SSE Composite Index and its sectoral index, the volatility variable is essentially the systematic cojumps of each index. For the SSE index, the three following stages are considered: The first stage was from March 14, 2013, to June 12, 2014; before the supply-side reform, the market volatility was in the normal range, and the additional factors affecting volatility were relatively few; the original cyclical characteristics of various 
TABLE 11: Descriptive statistics of cojumps covariance of the SSE Composite Index.

\begin{tabular}{|c|c|c|c|c|c|c|}
\hline $\operatorname{LOG}(+1)$ & MA & EL & FI & EN & $\mathrm{CO}$ & ME \\
\hline Mean & $4.90 e-05$ & $4.98 e-05$ & $4.22 e-05$ & $3.88 e-05$ & $3.31 e-05$ & $2.71 e-05$ \\
\hline Max & 0.005521 & 0.006014 & 0.005515 & 0.006397 & 0.006082 & 0.004531 \\
\hline Min & $-3.28 e-05$ & -0.000165 & -0.000260 & 0.000000 & $-7.65 e-05$ & -0.000122 \\
\hline Std. dev & 0.000300 & 0.000315 & 0.000262 & 0.000284 & 0.000264 & 0.000222 \\
\hline Skewness & 13.44008 & 12.50041 & 14.47060 & 16.02237 & 16.31177 & 16.20961 \\
\hline Kurtosis & 215.3063 & 191.3363 & 250.2250 & 307.8696 & 314.9918 & 298.9384 \\
\hline$J-B$ & $2192509^{*}$ & $1728078^{*}$ & $2966230^{*}$ & $4498925^{*}$ & $4711052^{*}$ & $4243188^{*}$ \\
\hline $\mathrm{ADF}$ & $-16.5461^{*}$ & $-13.9181^{*}$ & $-17.1582^{*}$ & $-16.1420^{*}$ & $-15.7680^{*}$ & $-14.7120^{*}$ \\
\hline $\mathrm{Q}(5)$ & $177.87^{*}$ & $189.16^{*}$ & $105.01^{*}$ & $81.107^{*}$ & $174.40^{*}$ & $102.94^{*}$ \\
\hline $\mathrm{Q}(10)$ & $253.77^{*}$ & $306.69^{*}$ & $116.53^{*}$ & $117.97^{*}$ & $196.89^{*}$ & $128.88^{*}$ \\
\hline $\mathrm{Q}(20)$ & $319.31^{*}$ & $418.01^{*}$ & $126.83^{*}$ & $127.83^{*}$ & $201.38^{*}$ & $141.30^{*}$ \\
\hline
\end{tabular}

Note: MA, EL, FI, EN, CO, and ME represent the logarithmic cojumps covariance of the SSE material, telecommunication, finance, energy, consumption, and medicine sector indices, respectively; ${ }^{*},{ }^{* *}$, and ${ }^{* * *}$ represent significance at the $1 \%, 5 \%$, and $10 \%$ levels, respectively.

industries are relatively clear. The second stage was from June 13, 2014, to January 26, 2016; although the supply-side reform did not occur, but the stock market entered an extreme situation where bulls and bears alternated, the degree of market volatility was very intense, and almost all sectors showed strong correlation and linkage with the broader market. The third stage was from January 27, 2016, to November 29, 2017; after the supply-side reform occurred, the policy influenced a massive reduction in production capacity in traditional surplus industries, and the shadow of the stock market crash still loomed; in a depressed economic environment, the original characteristics of some industry indices are likely to change accordingly.

From the correlation coefficients in Table 12, we can see that, in the first stage, the correlation coefficients of the three indices, material, financial, and energy, all reached over $80 \%$ with the general index, with the financial index reaching $92.4 \%$, while the consumer and medicine indices have relatively weak correlation with the general index; in particular the medicine index has a very low correlation with the general index. The difference in the degree of correlation at this stage can be observed visually from Figures 1-6. In the first stage, the indices of material, financial, and energy show a high correlation with the market and the trend of rising and falling, which has something to do with the economic periodicity of the industry. However, in the second stage, the trend of the three noncyclical industry indices, telecommunication, consumption, and medicine, which changes with the fluctuation of the market, has also become more obvious, which is obviously affected by the extreme market.

\subsection{Asymmetric Analysis of Systematic Cojumps on Volatility} Forecasts. Cyclical stocks are defined as stocks whose prices rise and fall with the rise and fall of the economic cycle. When the economy heats up, stocks in these sectors also rise rapidly; when the economy goes downhill, the prices of these stocks also fall. Corresponding to noncyclical stocks are some industries that provide the necessities of life and production. For this reason, variables that reflect the direction of stock market movements need to be set, and the directional symbols of systematic cojumps become key to reflecting this information.
According to Tables 13-15, the goodness of fit of the model after the sign decomposition of the systematic cojumps has improved to a large extent, and the following can also be found.

In the first stage, the JCOV- variables of the three indices of materials, financial, and energy have significantly positive influence coefficients, but the impact of the indices of telecommunications, consumption, and medicine is insignificant, because these industries are noncyclical sectors of the economy and the social demand for these products does not generally change much. As can also be seen in Table 12 of the correlation coefficients, the correlation coefficients of the three cyclical industry indices with the composite index are significantly higher than those of the noncyclical industry indices at this stage, and the correlation coefficient reflects the jump characteristics of volatility more adequately.

In the second stage, with the amplification of the abnormal volatility signal of the market, except for the telecommunications index, almost all indices have been affected by the stock market crash, and the volatility characteristics of some noncyclical industry indices have inevitably been changed by the impact of the market. Among them, the volatility of the financial and energy indices shows strongly cyclical characteristics in this stage, with both the JCOV+ and JCOV- impact coefficients being statistically significantly positive, implying that the prices of these two indices underwent corresponding jumps and volatile behavior with the ups and downs of the broader market, largely in line with the overall macroeconomic situation.

In the third stage, during the recovery period after the stock market crash, most of the indices regained their original attributes from the first stage, but the real economy is still in its infancy due to the impact of policy reforms, the financial indices are significantly less correlated with the broad market at this time, and the impact of systematic cojumps on future volatility is not obvious; meanwhile the original noncyclical sectors such as telecommunications have become new economic growth points along with raw materials and energy. Although noncyclical indices such as consumption and medicine also show high correlation coefficients between them and the broader market at this stage, the empirical results do not support their characteristics of cyclical sectors. 
TABLE 12: Table of correlation coefficients between various sector indices and SSE Composite Index returns.

\begin{tabular}{lcccccc}
\hline & Material & Telecommunication & Financial & Energy & Consumption & Medicine \\
\hline Phase I & 0.894 & -0.142 & 0.924 & 0.814 & 0.547 & -0.035 \\
Phase II & 0.959 & 0.929 & 0.963 & 0.879 & 0.975 & 0.946 \\
Phase III & 0.934 & 0.924 & 0.878 & 0.945 & 0.919 & 0.916 \\
\hline
\end{tabular}

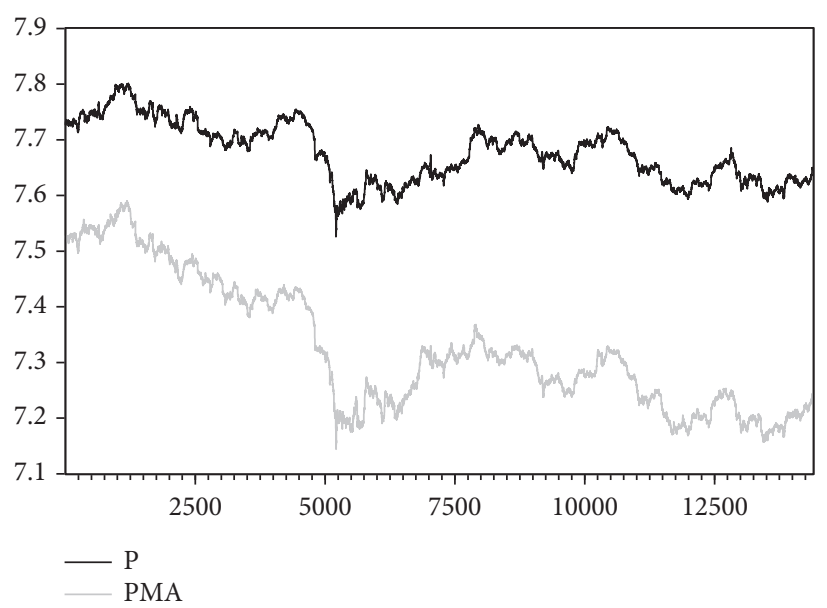

FIgure 1: Trend chart of SSE Composite Index (P) and material index (PMA).

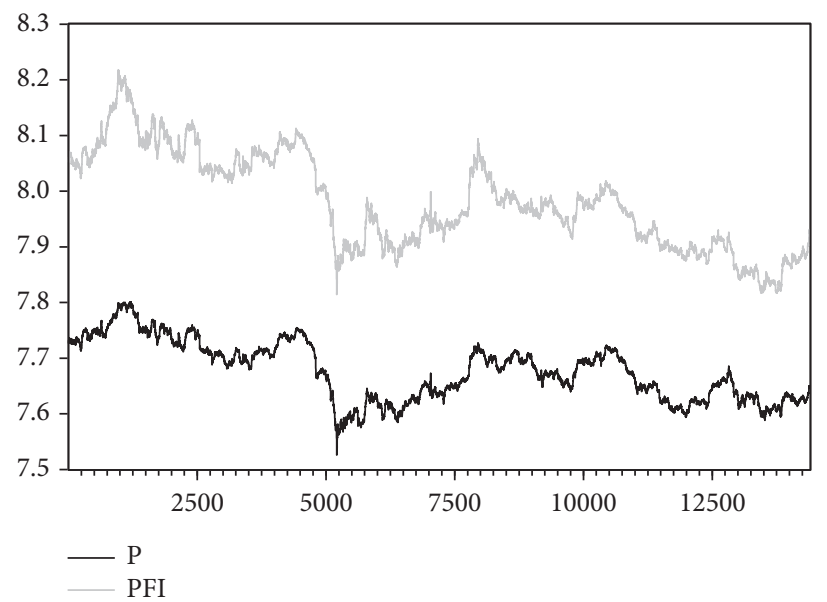

Figure 2: Trend chart of SSE Composite Index (P) and financial index (PFI).

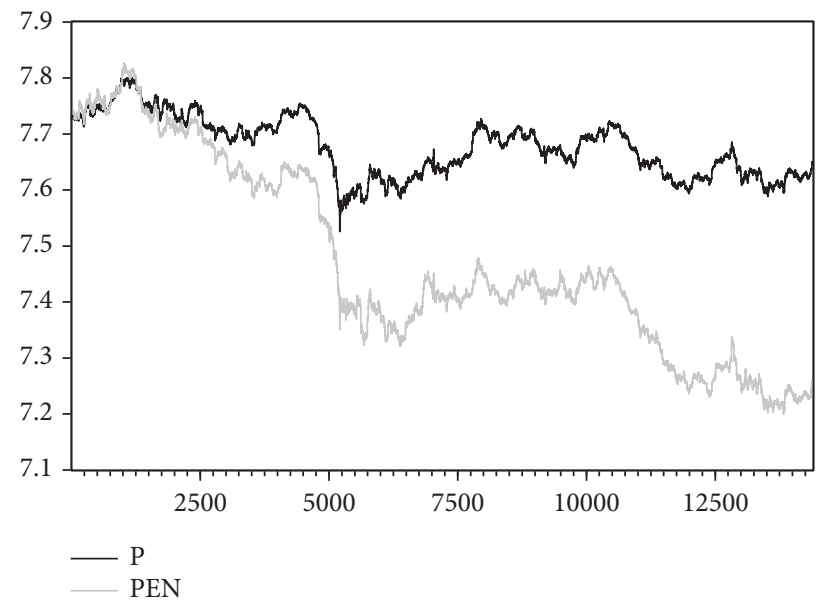

Figure 3: Trend chart of SSE Composite Index (P) and energy index (PEN). 


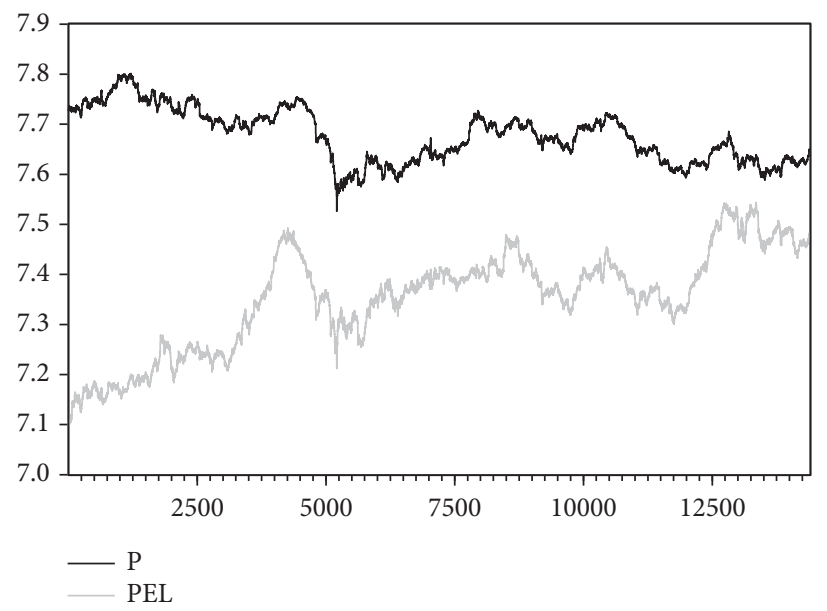

FIgURE 4: Trend chart of SSE Composite Index (P) and telecommunication index (PEL).

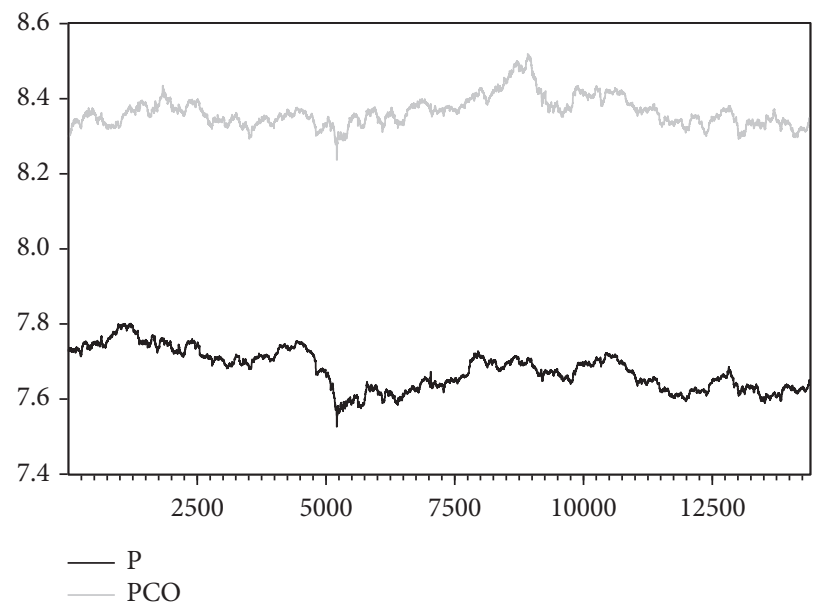

Figure 5: Trend chart of SSE Composite Index (P) and consumption index (PCO).

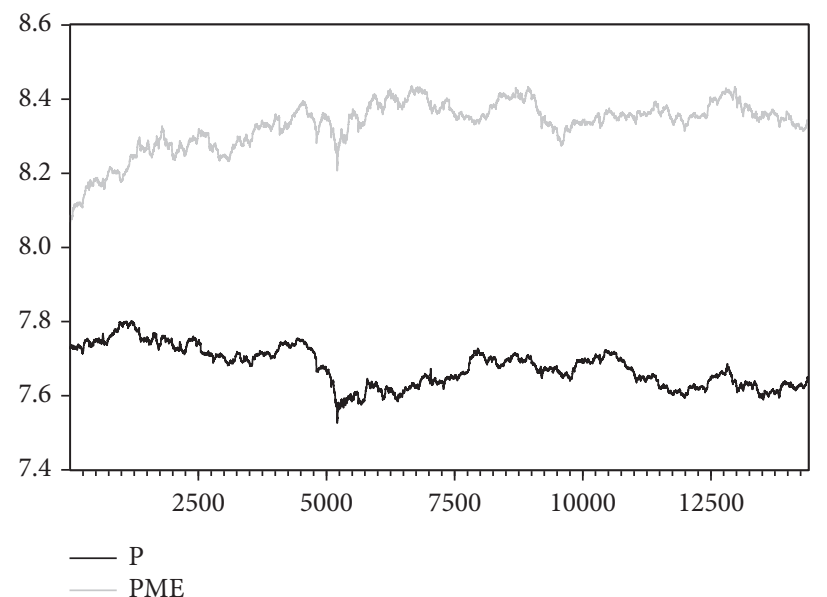

Figure 6: Trend chart of SSE Composite Index (P) and medicine index (PME).

According to the above analysis, the systematic cojumps of SSE sector index are of great significance to the prediction study of volatility, which reflects the degree of correlation between the broad market index and each sector index in different environments. In general, the systematic cojumps of cyclical industries have a significant positive impact on volatility in the next period, while the systematic cojumps of noncyclical industries have a significantly negative impact or 
TABLE 13: The estimation of the HAR-RV-JCOV-D model in the first stage.

\begin{tabular}{|c|c|c|c|c|c|c|c|}
\hline & $\alpha_{d}$ & $\alpha_{w}$ & $\alpha_{m}$ & $\alpha_{j_{1}}$ & $\alpha_{j_{2}}$ & $R^{2}(\%)$ & $R_{0}^{2}(\%)$ \\
\hline MJDMA & $0.2636^{*}(3.6540)$ & $5078^{*}(9.7369)$ & $0.0899(0.1527)$ & $1.9483^{* * *}(-1.9152)$ & $0.7678^{*}(3.0$ & 4.96 & 44.80 \\
\hline MJ & $0.3317^{*}(3.5180)$ & $4239^{*}(4.4116)$ & $-0.0609(-0.0$ & $54(0.0119)$ & $0.4677(0$ & 8.82 & 38.56 \\
\hline MJDFI & $0.2295^{* *}(2.4748)$ & $0.4672^{*}(5.4210)$ & $0.2524(0.3825)$ & $0.0570(0.0$ & $0.9819^{* * *}(1$ & 34.69 & 34.33 \\
\hline MJDEN & $-0.0347(-0.4804)$ & $0.6614^{*}(10.2063)$ & $1.8768^{*}(4.1064)$ & $1.3164(1.2010)$ & $1.0942^{* *}(2.4342)$ & 32.39 & 32.16 \\
\hline MJDCO & ** $(2.5$ & $9^{*}(5.5$ & 0.8043 & $1(-2$ & $20(-0$ & 36.80 & 36.58 \\
\hline MJDME & $0.4243^{*}(6.1018)$ & $0.4003^{*}(5.6306)$ & $-0.0828(-0.1580)$ & $-5.1360^{*}(-2.3703)$ & $0.2782(0.2486)$ & 50.85 & 50.29 \\
\hline
\end{tabular}

Note: MJDMA, MJDEL, MJDFI, MJDEN, MJDCO, and MJDME represent the HAR-RV-JCOV-D models for the SSE material, telecommunication, financial, energy, consumption, and medicine and other industry indices, respectively; $R_{0}^{2}$ represents the adjusted goodness of fit of the HAR-RV-JCOV models; ${ }^{*},{ }^{* *}$, and ${ }^{* * *}$ represent significance at the $1 \%, 5 \%$, and $10 \%$ levels.

TABLE 14: The estimation of the HAR-RV-JCOV-D model in the second stage.

\begin{tabular}{|c|c|c|c|c|c|c|c|}
\hline & $\alpha_{d}$ & $\alpha_{w}$ & $\alpha_{m}$ & $\alpha_{j_{1}}$ & $\alpha_{j_{2}}$ & $R^{2}(\%)$ & $R_{0}^{2}(\%)$ \\
\hline MJCMA & $0.5057^{*}(7.5926)$ & $0.3937^{*}(6.0369)$ & $-1.1981^{* *}(-2.2252)$ & $0.0519(0.1706)$ & $0.3595^{* *}(1.9676)$ & 73.33 & 73.18 \\
\hline MJCEL & $0.5333^{*}(7.4606)$ & $0.4023^{*}(5.9174)$ & $-1.8263^{*}(-3.2644)$ & $-0.2011(-0.7554)$ & $0.2102(1.2288)$ & 21 & 78.18 \\
\hline MJCFI & $0.3626^{*}(5.0397)$ & $0.5134^{*}(6.7341)$ & $0.1961(0.4974)$ & $0.3706^{* * *}(1.8131)$ & $0.6676^{*}(5.2742)$ & 72.40 & 71.73 \\
\hline MJCEN & $0.5272^{*}(7.1110)$ & $0.3677^{*}(4.8569)$ & $-0.5863(-1.1236)$ & *** (1.9110) & $0.4872^{*}(3.3704)$ & 74.68 & 74.15 \\
\hline MJCCO & $0.4121^{*}(5.3987)$ & $0.5012^{*}(6.5264)$ & $-0.7491(-1.4306)$ & $-0.1370(-0.7383)$ & $0.3288(1.4910)$ & 74.45 & 74.37 \\
\hline MJCME & $0.3914^{*}(5.9873)$ & $0.5147^{*}(6.9847)$ & $-0.5592(-1.9154)$ & $0.4475(0.9214)$ & $0.5704^{*}(3.4669)$ & 75.43 & 75.09 \\
\hline
\end{tabular}

Note: MJDMA, MJDEL, MJDFI, MJDEN, MJDCO, and MJDME represent the HAR-RV-JCOV-D models for the SSE material, telecommunication, financial, energy, consumption, and medicine and other industry indices, respectively; $R_{0}^{2}$ represents the adjusted goodness of fit of the HAR-RV-JCOV models; ${ }^{*}$, ${ }^{* *}$, and ${ }^{* * *}$ represent significance at the $1 \%, 5 \%$, and $10 \%$ levels.

TABLE 15: The estimation of the HAR-RV-JCOV-D model in the third stage.

\begin{tabular}{|c|c|c|c|c|c|c|c|}
\hline & $\alpha_{d}$ & $\alpha_{w}$ & $\alpha_{m}$ & $\alpha_{j_{1}}$ & $\alpha_{j_{2}}$ & $R^{2}(\%)$ & $R_{0}^{2}(\%)$ \\
\hline MJDMA & $854^{*}$ & $2604 *(3.8695)$ & $.6640 *(-2.8863)$ & $2.4777^{*}(2.7443)$ & $-0.4814(-0.9827)$ & .77 & .49 \\
\hline MJ & $51^{*}(7.6652)$ & $8^{*}(4.5498)$ & $-1.7786^{* *}(-2.4074)$ & $2.9781 *(2.1$ & 54( & & 60.53 \\
\hline & $4397^{*}(6.0500)$ & $82 *(5.3187)$ & *** $(-1.9404)$ & $2.0081(1.6$ & $8^{* * *}($ & 2 & 50.33 \\
\hline MJDEN & $5362 *(7.9895)$ & $2801^{*}(4.3758)$ & $-1.4226^{* *}(-2.3002)$ & $-0.8834^{* *}(-2.3276)$ & $4.0029^{*}(2.6$ & 52.04 & 51.33 \\
\hline MJDCO & $5976^{*}(8.52$ & $2022^{*}(2.84$ & $2(-2$ & $32(1.0$ & $57(-1$. & 4 & 51.29 \\
\hline MJDME & $0.5800^{*}(8.1133)$ & $0.2778^{*}(4.0144)$ & $-2.5362^{*}(-3.3592)$ & $2.1801(1.3794)$ & $-1.2389^{* *}(-2.0832)$ & 63.33 & 63.13 \\
\hline
\end{tabular}

Note: MJDMA, MJDEL, MJDFI, MJDEN, MJDCO, and MJDME represent the HAR-RV-JCOV-D models for the SSE material, telecommunication, financial, energy, consumption, and medicine and other industry indices, respectively; $R_{0}^{2}$ represents the adjusted goodness of fit of the HAR-RV-JCOV models; ${ }^{*}$, ${ }^{* *}$, and ${ }^{* * *}$ represent significance at the $1 \%, 5 \%$, and $10 \%$ levels.

insignificant impact; at the same time, the prediction of systematic cojumps on volatility is more influenced by the supply-side reform policy, and while the cyclical characteristics of individual industries disappear after the reform, some noncyclical industries become new support points and show the same pattern of ups and downs as the broad market.

\section{Conclusions and Recommendations}

Based on the nonparametric joint test and HAR forecasting model, this paper investigates the asymmetry of macro information shocks on volatility forecasting from the perspective of cojumps, using the high-frequency returns of the CSI 300 stock index and futures index, as well as the SSE Composite Index and industry index, and applies it to the forecasting of systematic risk. The main conclusions are as follows.

First, the prediction of cojumps on volatility exhibits significant asymmetry and interval reversal. On the one hand, in the post-supply-side reform stock index market, the asymmetry of volatility reverses to negative cojumps having less impact than positive cojumps; on the other hand, after the market switches from bull market to bear market, the asymmetry reverses to positive cojumps having less impact than negative cojumps. In the bull market, there is evidence of spillover of stock index to futures index market, which is mainly in the form of positive jumps.

Second, the symbolic cojumps covariance is a new estimator constructed based on signed jumps, and the interpretation of volatility by signed cojumps also has a significant asymmetric property. The signed cojumps estimator not only improves the in-sample fitting accuracy of the HAR model but also improves its out-of-sample prediction ability and thus improves the forecasting of volatility.

Third, the prediction of systematic cojumps on volatility is strongly influenced by supply-side reform policies. Before the reform, the systemic cojumps of cyclical industries had a significantly positive impact on the volatility of the next period, while the systemic cojumps of noncyclical industries had a negative or no obvious impact. After the reform, the cyclical characteristics of individual industries disappeared, 
while some noncyclical industries gradually became new economic growth points.

The conclusions of this paper have important significance for risk identification and long-term stable operation of financial markets. First, since cojumps risks are often difficult to hedge through derivatives, the direction of macro shocks should be identified and managed in an integrated manner when establishing an early warning mechanism for financial risks, and the risk forecasting and management system should be further improved. Second, systemic cojumps have a significant impact on volatility forecasts and have industry-specific and phase-specific effects. Investors should fully consider the linkage effect between markets when establishing hedging strategies and undertaking operations and adjust their decisions in a timely manner to achieve risk avoidance and increase returns according to the different impacts of cojumps on volatility under information shocks. Finally, in order to maintain the long-term stable operation of the financial market in the process of financial system reform and product innovation, regulators can identify potential sources of risk through forecasting volatility, make full use of the advantages of high-frequency information in the market, and analyze the characteristics of risk spillover and provide reference for systemic risk monitoring to achieve the long-term stable development of the domestic financial market.

\section{Data Availability}

The data used to support the findings of this study are available from the corresponding author upon request.

\section{Conflicts of Interest}

The authors declare that they have no conflicts of interest.

\section{Acknowledgments}

This work was supported by the National Natural Science Foundation of China "Research on the micro mechanism and governance of liquidity spiral in extreme stock market volatility" (71873023) and Science and Technology Project of Chongqing Education Commission "Research on the cojumps of asset prices and its prediction of high frequency volatility" (KJQN202001221).

\section{References}

[1] O. E. Barndorff-Nielsen and N. Shephard, "Power and bipower variation with stochastic volatility and jumps," Journal of Financial Econometrics, vol. 2, no. 01, pp. 1-37, 2004.

[2] D. Gilder, M. B. Shackleton, and S. J. Taylor, "Cojumps in stock prices: empirical evidence," Journal of Banking \& Finance, vol. 40, pp. 443-459, 2014.

[3] H. Wang, M. Yue, and H. Zhao, "Cojumps in China's spot and stock index futures markets," Pacific-Basin Finance Journal, vol. 35, pp. 541-557, 2015.

[4] Y. Tang and X. Lin, "Volatility modeling in consideration of the cojumps: based on the perspective of high-frequency data," Chinese Journal of Management Science, vol. 23, no. 8, pp. 46-53, 2015.

[5] H. Qu and P. Ji, "The role of cojumps in forecasting covariance matrices in Chinese stock markets: a study based on the multivariate HAR model," Journal of Management Science, vol. 29, no. 6, pp. 28-38, 2016.

[6] H. M. Zuo and Z. T. Liu, "Jump risk measurement and its Application in risk-return relationship test," Journal of Financial Research, no. 10, pp. 170-183, 2011.

[7] H. Zhao and K. J. Qin, "Jumps in stock prices and macro information release," Statistical Research, vol. 31, no. 4, pp. 79-89, 2014.

[8] A. J. Patton and K. Sheppard, "Good volatility, bad volatility: signed jumps and the persistence of volatility," The Review of Economics and Statistics, vol. 97, no. 03, pp. 683-697, 2015.

[9] W. Pu, Y. Chen, and F. Ma, "Forecasting the realized volatility in the Chinese stock market: further evidence," Applied Economics, vol. 48, no. 33, pp. 3116-3130, 2016.

[10] F. Ma, Y. Wei, and D. S. Huang, "Forecasting the realized volatility based on the signed return and signed jump variation," Journal of Management Sciences in China, vol. 20, no. 10, pp. 31-43, 2017.

[11] T. Bollerslev, T. H. Law, and G. Tauchen, "Risk, jumps, and diversification," Journal of Econometrics, vol. 144, no. 01, pp. 234-256, 2008.

[12] H. Zhou and J. Q. Zhu, “An empirical examination of jump risk in asset pricing and volatility forecasting in China's equity and bond markets," Pacific-Basin Finance Journal, vol. 20, no. 5, pp. 857-880, 2012.

[13] Z. H. Jian and C. Y. Li, "Systematic jumping risk and timevarying features of beta," Chinese Journal of Management Science, vol. 21, no. 3, pp. 20-27, 2013.

[14] J. Y. Liu, C. Y. Li, and Z. H. Jane, "Systematic jumps, heterogeneity jumps and tail characteristics," Systems Engineering-Theory \& Practice, vol. 35, no. 1, pp. 49-56, 2015.

[15] J. Lahaye, S. Laurent, and C. J. Neely, "Jumps, cojumps and macro announcements," Journal of Applied Econometrics, vol. 26, no. 6, pp. 893-921, 2011.

[16] O. E. Barndorff-Nielsen and N. Shephard, "Econometrics of testing for jumps in financial economics using bipower variation," Journal of Financial Econometrics, vol. 4, no. 01, pp. 1-30, 2006.

[17] F. Corsi, A Simple Long Memory Model of Realized volatility, pp. 1-28, University of Lugano, Lugano, Switzerland, 2004.

[18] F. Audrino, F. Sigrist, and D. Ballinari, "The impact of sentiment and attention measures on stock market volatility," International Journal of Forecasting, vol. 36, no. 2, pp. 334357, 2020.

[19] X. Huang, J. Liu, X. Zhang, and Y. Zhu, "Volatility premium and term structure of China blue-chip index options," Emerging Markets Finance and Trade, vol. 56, no. 3, pp. 527-542, 2020.

[20] R. Chen, H. Chen, C. Jin, B. Wei, and L. Yu, "Linkages and spillovers between internet finance and traditional finance: evidence from China," Emerging Markets Finance and Trade, vol. 56, no. 6, pp. 1196-1210, 2020. 